\title{
Acid-Base Equilibrium Constants for the Reaction of Tribenzylamine with Picric Acid and with Trinitro-m- Cresol in Benzene, from Spectrophotometric Data
}

\author{
By Marion Maclean Davis and E. Anne McDonald
}

\begin{abstract}
The relative acidic strengths of picric acid and trinitro- $m$-cresol in benzene have been measured spectrophotometrically in terms of their reactivity with the base, tribenzylamine. The respective constants found for the combination of tribenzylamine with picric acid and with trinitro- $m$-cresol in benzene at $25^{\circ} \mathrm{C}$ are $1.58 \times 10^{3}$ and $4.48 \times 10^{2}$. The same relative order of strengths would be predicted on theoretical grounds. The constant for picric acid is in close agreement with previous measurements of the dielectric constant of benzene solutions of tribenzylammonium picrate. The method used for assessment of the relative strengths of the two acids is superior to measurements of their ionic dissociation in water.
\end{abstract}

\section{Introduction}

During the first quarter of this century, the definitions of an acid and a base most commonly taught and applied were founded on the behavior of aqueous solutions. As is well known, acidic and basic properties were referred, respectively, to hydrogen and hydroxyl ions, numerous investigators undertook the measurement of the ionic dissociation constants of acids and bases in water, and the $\mathrm{pH}$ scale came into widespread use as a means of expressing relative acidities and basicities. The currently popular Brønsted-Lowry proton-transfer theory of acidity and basicity has clarified the role of the solvent. ${ }^{1}$ It is now recognized that water can function both as a base and as an acid, and this, of course, is the reason a continuous $\mathrm{pH}$ scale for aqueous solutions can exist. Moreover, the effect of other solvents on dissolved acids and bases is now to some degree predictable, because the electronic structure of a molecule reveals whether it can serve as a proton donor, as a proton acceptor, or

\footnotetext{
1 The authors do not reject the much broader Lewis concept of acidity, but it offers no advantage over the Brønsted-Lowry concept when hydrogen acids only are considered, as in this paper. Furthermore, acid-base reactions that involve the formation of a hydrogen bridge do not entirely parallel reactions in which a coordinate covalent bond is formed between the acid and the base.
}

as both donor and acceptor. But whenever the solvent displays acidic or basic character, the nature of the reaction between a dissolved acid and base is partially masked. The ideal condition for studying the reaction between an acid and a base would be to use no solvent at all. The nearest practicable approach to such a condition, when organic acids and bases are concerned, is to use an inert or "aprotic" solvent; that is, a solvent that can neither add nor release protons. Benzene and carbon tetrachloride are examples of aprotic solvents that are suitable for such studies. At one time many proponents of the ionization theory assumed, from an overemphasis of the role of ions in chemical reactions and inadequate experimental evidence, that acidbase reactions do not occur in any medium of such low dielectric constant. Subsequently, Hantzsch, Brønsted, LaMer, and others demonstrated that instantaneous acid-base reactions occur as readily in an aprotic medium as in an ionizing medium. However, widespread awareness of their work does not yet exist, acid-base reactions in aprotic media remain a neglected field of study, and a general tendency persists to employ, in analytical procedures and as media for reactions, only solvents that promote ionization.

In two previous publications from this Bureau 
$[1,2],{ }^{2}$ some of the earlier studies of acid-base reactions in aprotic media were reviewed, and new experimental data, both qualitative and quantitative, were presented. The method employed by us was to study, by means of the spectrophotometer, the reaction between different acidic indicator dyes and various organic bases in benzene and other organic solvents. Of particular interest was the discovery that organic bases of various types-for example, primary, secondary, and tertiary aliphatic amines-exhibit specific differences in their behavior with acids in an aprotic solvent, such as benzene. These differences are masked in an amphiprotic solvent, like water. Other studies still in progress provide additional evidence of the specificity of the behavior of bases and of acids in inert solvents. Such experiments point to the need for further investigations of acid-base reactions in inert solvents, as a prerequisite both to the development of methods for the measurement of acidity and basicity in such media and to a better understanding of acid-base relationships in water and other "active" solvents.

This paper deals with the reaction of tribenzylamine with picric acid and with trinitro- $m$-cresol in benzene. The acid-base equilibrium constants of the reactions were determined spectrophotometrically. As will be shown, the results are in agreement with conclusions arrived at from other experimental procedures.

\section{Equipment and Procedure}

The spectrophotometric equipment used was the same as that described in the preceding paper of this series [2], except for changes designed for protection of the solutions from the heating effect of the light source. The cell compartment was replaced by a new box with double walls at the bottom, sides, and top. In the space between the two walls, water from a constant-temperature bath circulates continuously. The temperature of the bath is maintained at $25.0^{\circ} \pm 0.1^{\circ} \mathrm{C}$, and the contents of the cell can be kept at a temperature not more than $0.2^{\circ} \mathrm{C}$ above that of the bath. The light source was also moved about 1 in. farther away from the cell compartment.

The procedure followed in the preparation and

\footnotetext{
${ }^{2}$ Figures in brackets indicate the literature references at the end of this paper.
}

dilution of solutions and in the recording of data has already been described [1,2]. In some of the measurements, one cell contained the pure solvent and the other the solution under investigation. However, when working with a considerable excess of tribenzylamine in the solution, an equivalent amount of tribenzylamine was placed in the reference cell. Such cases will be noted in section IV.

The symbols and terminology used are as follows: $T_{s}$ (transmittancy of the solute $)=T_{\text {soln }} / T_{\text {solv }}$; $a_{s}$ (absorbancy or optical density of the solute) $=$ $-\log { }_{10} T_{s} ; a_{M}$ (molar absorbancy of the solute)= $a_{s} /(b \times M) ; b=$ length in centimeters of the absorption cell; $M=$ molar concentration of the solution [3].

\section{Materials}

Benzene.-The high grade of commercial benzene used in previous work became unavailable while these investigations were in progress. However, benzene from another source was found to be equally satisfactory when dried over Drierite and then redistilled [4].

Picric acid.-A practical grade of picric acid, containing 10 percent of water, was recrystallized twice from boiling water, dried in a vacuum oven at $100^{\circ} \mathrm{C}$, then precipitated from benzene solution by cyclohexane, and finally dried in a vacuum oven at $80^{\circ} \mathrm{C}$. The melting point was $122.1^{\circ}$ to $122.3^{\circ} \mathrm{C}^{3}$

Tribenzylamine.-A high grade of commercial tribenzylamine was recrystallized from 95-percent ethyl alcohol and then dried in a vacuum oven at $80^{\circ} \mathrm{C}$. The melting point was $92.0^{\circ}$ to $92.1^{\circ} \mathrm{C}$.

Trinitro-m-cresol. - A high grade of commercial trinitro- $m$-cresol was recrystallized once from 95percent ethyl alcohol and twice from benzene. After drying in a vacuum oven at $80^{\circ} \mathrm{C}$, the melting point was $107.0^{\circ}$ to $107.2^{\circ} \mathrm{C}$.

\section{Data and Calculations}

Reaction of tribenzylamine with picric acid.-In the pure, dry state, tribenzylamine and picric acid are colorless solids and give colorless solutions in benzene. When solutions of the acid and the base are mixed, an instantaneous reaction occurs, as

${ }^{3}$ Melting points were determined in an electrically heated bath containing silicone oil, with an ASTM thermometer for 3-in. immersion. 
shown by the appearance of a yellow color. ${ }^{4}$ The reaction was studied quantitatively by measurement of the spectral transmittancy of solutions that contained a constant amount of picric acid mixed with varying amounts of the amine. A series of transmittancy curves obtained for such solutions are presented in figure 1 . Curve 0 is for

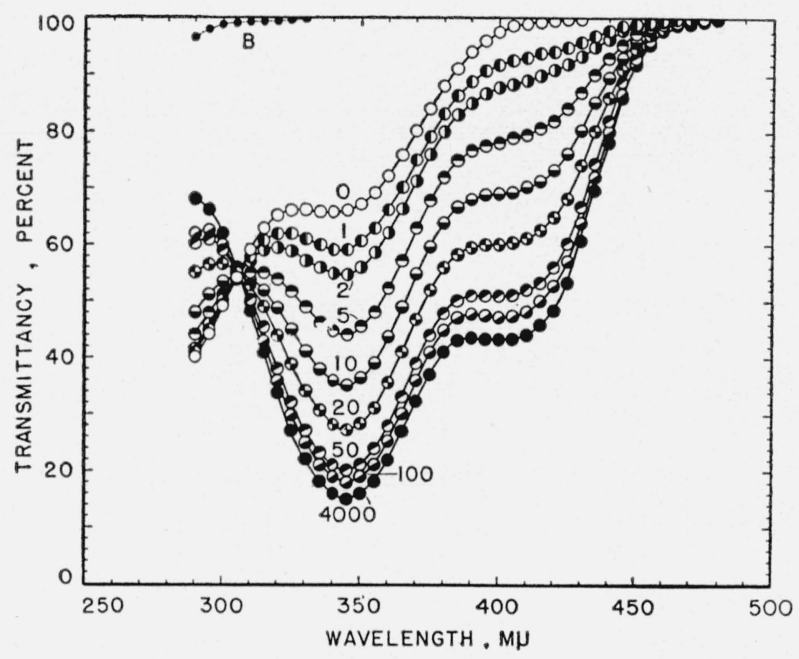

FIgURE 1. Transmittancy curves for picric acid, for tribenzylamine, and for their mixtures in benzene (1-cm cell).

0 , Picric acid, $5 \times 10^{-5}-M_{;}$(1) to $(4,000), 5 \times 10^{-5}-M$ picric acid with from 1 to 4,000 molar equivalents of tribenzylamine; $\mathrm{B}$, tribenzylamine, $5 \times 10^{-3}-M$.

$5 \times 10^{-5}-M$ picric acid in benzene, and the curves designated 1 through 4,000 are for solutions that contained the same concentration of picric acid mixed with from 1 to 4,000 molar equivalents of tribenzylamine. In the case of the curve marked 4,000 , the reference cell contained 4,000 molar equivalents of tribenzylamine; in all other cases, it contained benzene only. The change produced by 4,000 molar equivalents of tribenzylamine was no greater than that produced by 2,000 molar equivalents of the base, and the curve marked 4,000 is therefore the limiting curve for the reaction. ${ }^{5}$ Measurements cannot be made for

\footnotetext{
4 The presence of traces of water adsorbed from the air by any of the materials or glassware also produces a yellow color. It is therefore necessary to take every possible precaution to exclude moisture and to prepare fresh stock solutions of the materials at frequent intervals. Little difficulty is experienced if measurements are performed in cool, dry weather.

${ }^{5}$ In the preparation of solutions that contained 2,000 or 4,000 molar equiva lents of tribenzylamine, the slight evaporation of solvent upon exposure of the $0.25-M$ stock solution to the air caused some of the base to be deposited on the surface of the flasks and pipettes. The actual concentration of tri benzylamine was therefore not quite 2,000 or 4,000 molar equivalents. However, it was evident that a further increase in concentration would not produce a measurable change in transmittancy.
}

wavelengths shorter than $280 \mathrm{~m} \mu$, because of the high absorbancy of the solvent.

A benzene solution of tribenzylamine more dilute than $10^{-3}-M$ shows almost complete transmittancy at wavelengths greater than $300 \mathrm{~m} \mu$. More concentrated solutions exhibit measurable absorbancy at wavelengths shorter than about $350 \mathrm{~m} \mu$, as indicated by the transmittancy curve for $5 \times 10^{-3}-M$ tribenzylamine (curve B, fig. 1). Determination of the applicability of Beer's law is not easy, because of the steepness of the transmittancy curves for the higher concentrations of the base. However, in the range $5 \times 10^{-4}-M$ to $5 \times 10^{-2}-M$, the values for molar absorbancies agreed within experimental error, and there is no reason for expecting the molar absorbancy to show any effect of concentration. It is not possible to determine with certainty the change in transmittancy that occurs when tribenzylamine is converted to the tribenzylammonium group, but it is reasonable to assume that the change is inappreciable for a $5 \times 10^{-5}-M$ solution, even at the shortest wavelengths.

The curve for picric acid shows a shallow transmission band near $325 \mathrm{~m} \mu$ and a shallow absorption band near $340 \mathrm{~m} \mu$. At the concentration employed, there was no measurable absorbancy at wavelengths greater than about $430 \mathrm{~m} \mu$. Molar absorbancies for $5 \times 10^{-5}-M$ and $5 \times 10^{-4}-M$ solutions were the same within experimental error. Picric acid has been shown by cryoscopic measurements to exist in the monomeric state in benzene [5], and infrared studies indicate a hydrogen bridge between the phenolic oxygen and the nitro group in ortho-substituted phenols [6]. Conformity of its benzene solution to Beer's law is therefore to be anticipated.

The transmittancy curve for tribenzylammonium picrate in benzene shows a fairly deep absorption band near $342 \mathrm{~m} \mu$ and a very shallow band near $410 \mathrm{~m} \mu$. Curves 0 through 10 in figure 1 show a well-marked isosbestic point at approximately $304.5 \mathrm{~m} \mu$. With concentrations of tribenzylamine greater than 10 molar equivalents, the isosbestic point was shifted to about $305.5 \mathrm{~m} \mu$.

Solutions of tribenzylammonium salts are all colorless if the acidic component is colorless, whereas all picrates give yellow solutions. The absorption bands that give rise to the yellow color of tribenzylammonium picrate are therefore attributable to a loosening of electrons in the 
$-\mathrm{OC}_{6} \mathrm{H}_{2}\left(\mathrm{NO}_{2}\right)_{3}$ group. This loosening is not so great as in quaternary ammonium picrates, because the transmittancy curves for the latter compounds in benzene are displaced appreciably toward longer wavelengths [7]. For a dilute solution of a quaternary ammonium picrate in benzene, for example, tetraethylammonium picrate, the studies of Kraus and his collaborators indicate the structure $\mathrm{Et}_{4} \stackrel{+}{\mathrm{N}} \overline{\mathrm{P}}$. This formula signifies an ion pair, held together by Coulombic attraction. The formula for tribenzylammonium picrate, $\left(\mathrm{C}_{6} \mathrm{H}_{5} \mathrm{CH}_{2}\right)_{3} \stackrel{+}{\mathrm{N}} \mathrm{H} \ldots . \overline{\mathrm{O}} \mathrm{C}_{6} \mathrm{H}_{2}\left(\mathrm{NO}_{2}\right)_{3}$, accounts for the difference in the spectral absorption of the two salts. It is also in harmony with the views of Kraus, who wrote: "When a partially substituted ammonium salt is dissolved in a solvent medium the molecules of which have no affinity for the proton, interaction takes place between the negative ion and the proton of the positive ion. In other words, there is formed what is commonly known as a hydrogen bond, the energy of rupture of which may be much higher than the energy necessary to overcome the Coulombic forces acting between the ions [8]." The conclusion cited was derived from measurements of conductance.

The reaction under discussion may be formulated quite simply as the union of one molecule of tribenzylamine with one molecule of picric acid to give a molecule of tribenzylammonium picrate. The hydrogen bond (or bridge) is generally much weaker than a covalent bond. A rupture of the hydrogen bridge in tribenzylammonium picrate can occur in two places. If the break comes between nitrogen and hydrogen, the products are tribenzylamine and picric acid. A break between hydrogen and oxygen gives tribenzylammonium and picrate ions. Experimental data indicate that appreciable dissociation of the salt into tribenzylamine and picric acid occurs in benzene solution. Spectrophotometric measurements furnish no evidence of ionic dissociation. The possibility of extremely minute concentrations of ions, is not excluded, however. Kraus and Fuoss, from measurements of conductance, calculated for tetraisoamylammonium picrate and for triisoamylammonium picrate in benzene the respective ionic dissociation constants, $8.9 \times 10^{-18}$ and $2.5 \times 10^{-21}$ at $25^{\circ} \mathrm{C}[9,10]$. These values correspond to an ionic dissociation of approximately a millionth of a percent in the case of tetraisoamylammonium picrate, and approximately a billionth of a percent in the case of triisoamylammonium picrate. The ionic dissociation of tribenzylammonium picrate in benzene has not been measured, but it probably does not differ greatly from that of triisoamylammonium picrate.

If, now, the equation for this reaction is written in the form,

$$
\underset{\text { (acid) }}{A}+\underset{\text { (base) }}{B} \rightleftharpoons \underset{\text { (salt) }}{S}
$$

the association constant is expressed according to the familiar law of mass action as

$$
K_{a s s o c}=[S] /\{[A][B]\} .
$$

The value obtained for $K_{\text {assoc. }}$ will be a measure of the acidic strength of picric acid and of the basic strength of tribenzylamine. In order to compute the values of $[S],[A]$, and $[B]$ for various mixtures, it is necessary to know the initial concentrations $C_{a}$ and $C_{3}$ of the acid and base, respectively, and the values of $a_{s}$ for the acid, for the salt, and for mixtures of the two, at one or more wavelengths. The quotient, $\left[a_{s}\right.$ (mixture) $-a_{s}$ (acid) $] /\left[a_{s}\right.$ (salt) $a_{s}$ (acid)], gives the fraction of the acid converted to the salt. This fraction multiplied by the initial concentration of the acid $\left(5 \times 10^{-5}-M\right)$ gives $[S]$, the molar concentration of salt in the mixture. The concentrations of acid and base in the equilibrium mixture are, respectively, $C_{a}-[S]$ and $C_{b}-[S]$. Experimental values of $a_{s}$ for the acid, salt, and 19 mixtures of the two at the wavelength $410 \mathrm{~m} \mu$ are given in table 1, together with the calculated values for $[S],[A],[B]$, and $K_{a s s o c}$. Values of $K_{\text {assoc. }}$ computed for data at $342 \mathrm{~m} \mu$ are also included. The precision of the data is obviously less for mixtures that contain very small quantities of the acid or the salt. The average of 16 intermediate values for $K$ is $1.58 \times 10^{3}$ for measurements at $410 \mathrm{~m} \mu$ and $1.52 \times 10^{3}$ for measurements at $342 \mathrm{~m} \mu$. Because of uncertainty as to a possible contribution from basic components to the absorbancy at $342 \mathrm{~m} \mu$, the value obtained for $410 \mathrm{~m} \mu$ is probably the more reliable one.

The manner in which the fraction of picric acid converted to tribenzylammonium picrate varies with $C_{b}$ is shown in figure 2 , curve 1 . The open circles represent values for $410 \mathrm{~m} \mu$ and the solid circles values for $342 \mathrm{~m} \mu$. The change produced 
TABLE 1. Experimental data and association constant for the reaction of tribenzylamine and picric acid in benzene

\begin{tabular}{|c|c|c|c|c|c|c|}
\hline \multirow{3}{*}{$\begin{array}{l}\text { Initial con- } \\
\text { centration } \\
\text { of base } \mathrm{s}\end{array}$} & \multicolumn{6}{|c|}{ From measurements at- } \\
\hline & \multicolumn{5}{|c|}{$410 \mathrm{~m} \mu$} & \multirow{2}{*}{$\frac{342 \mathrm{~m} \mu}{K \times 10^{-3}}$} \\
\hline & $a_{*}$ & {$[S] \times 10^{5} \mathrm{~b}$} & {$[A] \times 10^{3 \mathrm{~b}}$} & {$[B] \times 10^{5} \mathrm{~b}$} & $K \times 10^{-3}$ & \\
\hline $0 \ldots$ & 0.0039 & & & & & \\
\hline $1 \ldots$ & .0311 & 0.3857 & 4.614 & 4. 614 & ${ }^{*} 1.81$ & ${ }^{*} 1.69$ \\
\hline $2 \ldots$ & .0501 & .6551 & 4. 345 & 9.345 & 1.61 & 1. 53 \\
\hline $4 \ldots$ & ๑. 0857 & 1.160 & 3.840 & 18.84 & 1.60 & 1.47 \\
\hline $5 \ldots$ & .1002 & 1. 366 & 3. 634 & 23.63 & 1. 59 & 1.57 \\
\hline $6 \ldots$ & c. 1135 & 1. 554 & 3. 446 & 28.45 & 1. 58 & 1. 53 \\
\hline $8 \ldots$ & c. 1355 & 1. 866 & 3.134 & 38.13 & 1. 56 & 1.45 \\
\hline $10 \ldots$ & .1537 & 2. 124 & 2.876 & 47.88 & 1. 54 & 1. 52 \\
\hline 12. & c. 1733 & 2. 402 & 2. 598 & 57.60 & 1.61 & 1. 52 \\
\hline $15 \ldots$ & .1938 & 2. 693 & 2. 307 & 72.31 & 1.61 & 1. 58 \\
\hline $16 \ldots$ & • 1993 & 2. 771 & 2. 229 & 77.23 & 1.61 & 1.51 \\
\hline $20 \ldots$ & .2140 & 2. 979 & 2. 021 & 97.02 & 1. 52 & 1.52 \\
\hline $25 \ldots$ & .2358 & 3. 288 & 1.712 & 121.7 & 1. 58 & 1.54 \\
\hline $30_{\ldots}$ & .2510 & 3. 504 & 1.496 & 146.5 & 1.60 & 1.48 \\
\hline $35 \ldots$ & .2604 & 3. 637 & 1.363 & 171.4 & 1.56 & 1.48 \\
\hline $40 \ldots$ & .2700 & 3. 773 & 1. 227 & 196. 2 & 1. 57 & 1.51 \\
\hline $50 \ldots$ & .2840 & 3. 972 & 1.028 & 246.0 & 1. 57 & 1.58 \\
\hline $100 \ldots$ & .3179 & 4. 453 & 0.547 & 495.5 & 1.64 & 1.49 \\
\hline $500 \ldots$ & .3382 & 4. 741 & .259 & 2,495 & $*_{0}^{*} 73$ & ${ }^{*} 0.63$ \\
\hline $1,000_{\text {_ }}$ & .3487 & 4. 889 & .111 & 4,995 & ${ }^{*} .88$ & *. 74 \\
\hline \multirow[t]{2}{*}{$\begin{array}{l}\text { Limiting } \\
\text { value. }\end{array}$} & d. 3565 & 5. 000 & & & & \\
\hline & & & & Average & อ 1.58 & $\bullet 1.52$ \\
\hline
\end{tabular}

${ }^{\text {a }}$ Expressed as molar equivalents of $5 \times 10^{-5}-M$ picric acid.

In moles per liter of solution.

- Average of 5 values.

a Observed value; calculated value is the same.

e Values marked with an asterisk are excluded from the average.

in the absorbancy is relatively great for small values of $C_{b}$. When the value of $C_{b}$ is greater than about 50 molar equivalents, much larger increments are necessary to cause measurable changes in the absorbancy and to determine the limiting value. For example, when $C_{b}=200$ or 500 molar equivalents, identical values of $a_{s}$ were obtained. Calculations of $K$ using this provisional limiting value showed a slight trend. Calculations were then made with various assumed limiting values for $a_{s}$. The value that gave the most consistent results was 0.3565 . The validity of this number was confirmed experimentally for solutions that contained $C_{b}=2,000$ and 4,000 molar equivalents, in which the minute residual quantities of picric acid are not detectable spectrophotometrically.

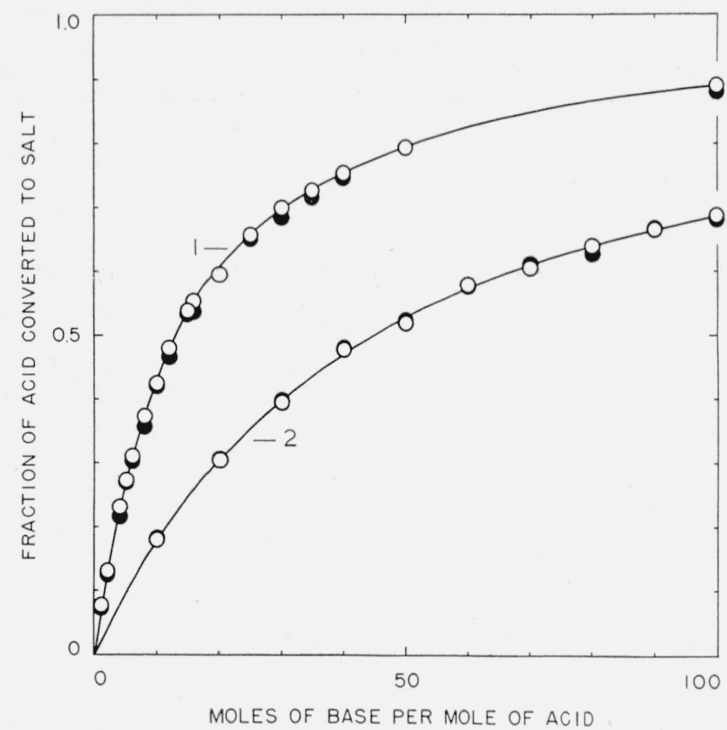

Figure 2. Effect of the concentration of tribenzylamine, expressed in molar equivalents, on the fraction of the acid converted to salt in benzene.

$1,5 \times 10^{-5}-M$ picric acid. Open circles, values for $410 \mathrm{~m} \mu$; solid circles, values for $342 \mathrm{~m} \mu$. $2,5 \times 10^{-5}-M$ trinitro- $m$-cresol. Open circles, values for $400 \mathrm{~m} \mu$; solid circles, values for $345 \mathrm{~m} \mu$.

Equation 2 may also be expressed in logarithmic terms as follows:

$$
\log [S] /[A]=\log [B]+\log K_{a s s o c} .
$$

Equation 3 has the form of the familiar equation for the straight line, $y=m x+b$, in which $m$ signifies the slope of the line and $b$ is its intercept on the $x$-axis. For the reaction postulated (eq. 1), $m$ should equal 1. In figure 3, for convenience, the values for $\log [S] /[A]$ and $-\log [B]$ are plotted along the vertical and horizontal axes, respectively. The slope then becomes negative in sign. The line shown in figure 3 was drawn with the theoretical slope, minus 1 . $\log K_{\text {assoc. }}$ is the intercept on the horizontal axis for $\log [S] /[A]=0$. It is evident from inspection of figure 3 as well as of table 1 that the experimental values showed no trend when the concentration of the base was varied but conformed closely to the equation assumed for the reaction. The validity of eq 1 is therefore supported by the experimental results as well as by the fact that any reaction other than the one postulated would be inconsistent with the properties of tribenzylamine and picric acid. 


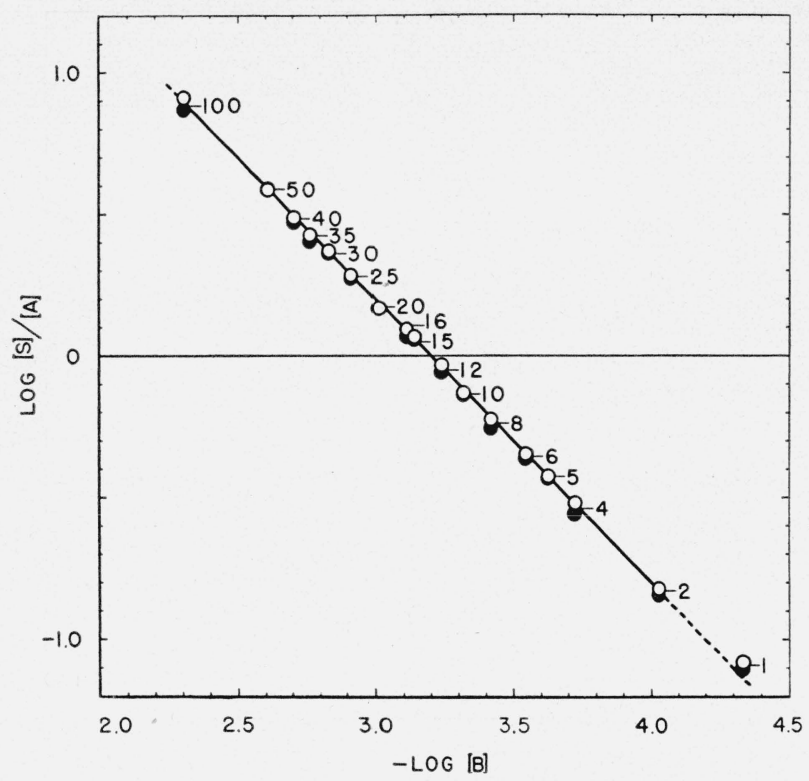

FIGURE 3. Graphical evaluation of association constant for the reaction of $5 \times 10^{-5}-M$ picric acid with tribenzylamine in benzene.

Experimental points marked 1 through 100 indicate the number of molar equivalents of tribenzylamine per mole of picric acid. Open circles, values for $410 \mathrm{~m} \mu$; solid circles, values for $342 \mathrm{~m} \mu$.

From the association constant, $1.58 \times 10^{3}$, it may be calculated that in a $5 \times 10^{-5}-M$ solution of tribenzylammonium picrate in benzene approximately 93 percent of the salt would be dissociated into tribenzylamine and picric acid at $25^{\circ} \mathrm{C}$.

Reaction of tribenzylamine with trinitro-m-cre$s o l$.-Trinitro- $m$-cresol (3-methylpicric acid) is a colorless solid and gives a colorless solution in benzene. The transmittancy curve for $5 \times 10^{-5}-M$ trinitro- $m$-cresol in benzene (fig. 4 , curve 0 ) resembles that of equimolar picric acid in benzene but is shifted slightly toward shorter wavelengths. Instead of a shallow absorption band near 340 $\mathrm{m} \mu$, there is a plateau in the region 325 to $335 \mathrm{~m} \mu$. No measurable absorbancy was observed for a $5 \times 10^{-5}-M$ solution at wavelengths greater than about $440 \mathrm{~m} \mu$. Deviations from Beer's law were not detected for solutions of four different concentrations in the range $5 \times 10^{-5}-M$ to $5 \times 10^{-4}-M$, and there is no reason to expect either association or dissociation of the acid in a benzene solution of the concentration used in the investigation.

Comparison of curves 1 and 2 in figure 2 reveals that a greater excess of tribenzylamine is required for the conversion of trinitrocresol to its tribenzylammonium salt in benzene than is required for

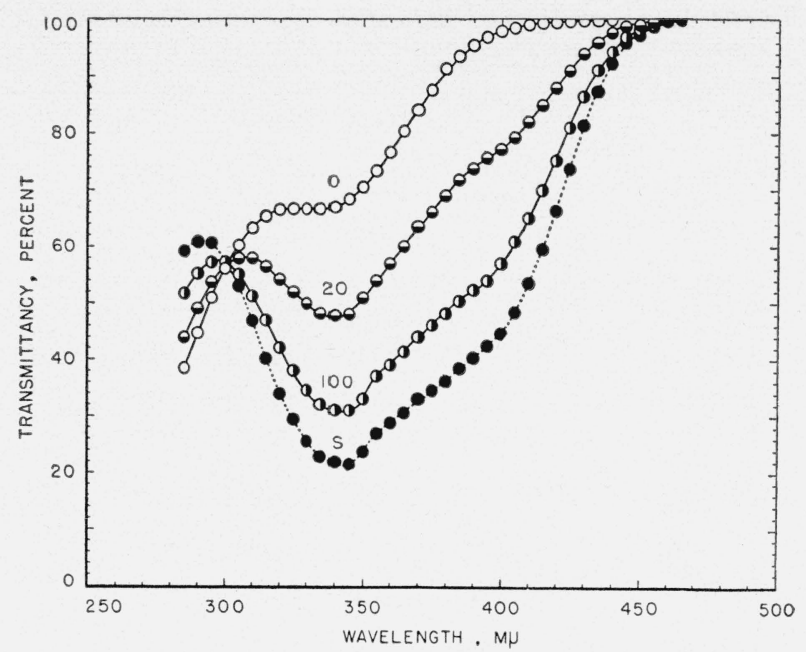

Figure 4. Transmittancy curves for trinitro-m-cresol and for its mixtures with tribenzylamine in benzene (1-cm cell).

0 , Trinitro- $m$-cresol, $5 \times 10^{-5}-M$; (20) and (100), $5 \times 10^{-5}-M$ trinitro- $m$-cresol, with 20 and 100 molar equivalents of tribenzylamine, respectively; $\mathrm{S}$, calculated limiting curve for the reaction of $5 \times 10^{-5}-M$ trinitro- $m$-cresol with tribenzylamine.

the conversion of picric acid to tribenzylammonium picrate. When about 2,000 molar equivalents of tribenzylamine were added to $5 \times 10^{-5}-M$ trinitrocresol, precipitation occurred. The limiting value was therefore not measurable, but it was calculated in the manner described for tri-

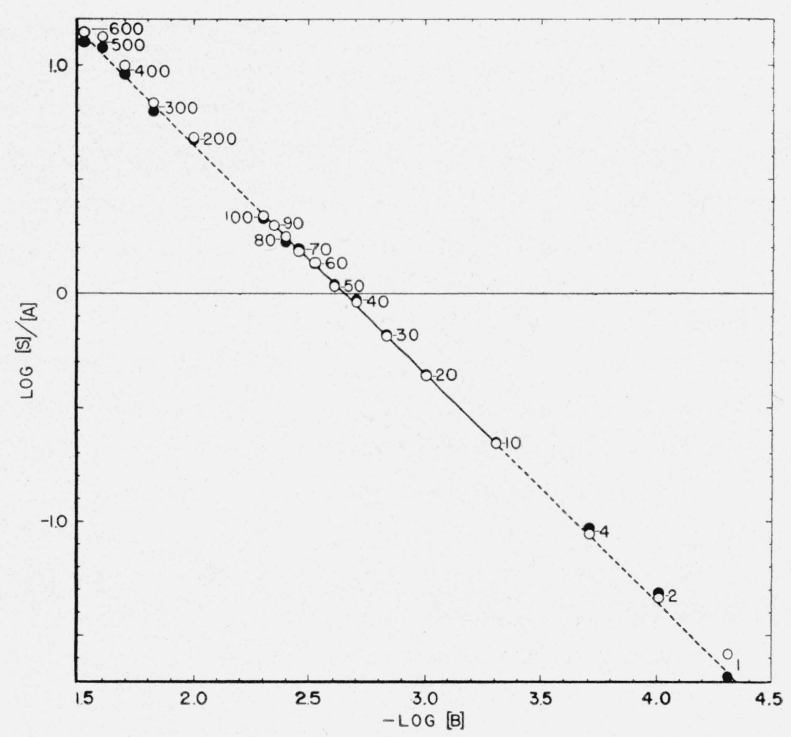

Figure 5. Graphical evaluation of association constant for the reaction of $5 \times 10^{-5}-M$ trinitro-m-cresol with tribenzylamine in benzene.

Experimental points marked 1 through 600 indicate the number of molar equivalents of tribenzylamine per mole of trinitro- $m$-cresol. Open circles, values for $400 \mathrm{~m} \mu$; solid circles, values for $345 \mathrm{~m} \mu$. 
benzylammonium picrate. The data presented in table 2 are for trinitro- $m$-cresol and 18 mixtures with tribenzylamine and correspond to those already discussed for mixtures of picric acid with tribenzylamine. Measurements at $400 \mathrm{~m} \mu$ and $345 \mathrm{~m} \mu$ gave the same average value, 448, for $K_{\text {assoc. }}$ From inspection of figure 5 it may be seen that all of the experimental values for log $[S] /[A]$ versus $-\log [B]$ fall on or very close to the theoretical curve. In view of the unlikelihood of a reaction other than the one postulated and the consistency of the results, the value 448 for $K_{\text {ossoc. }}$ may be accepted with confidence. From this result it may be computed that in $5 \times 10^{-5}-M$ benzene solution, the tribenzylammonium salt of trinitro- $m$-cresol would be 98 percent dissociated into tribenzylamine and trinitro- $m$-cresol at $25^{\circ} \mathrm{C}$.

Although it was not possible to measure the transmittancy curve for the tribenzylammonium salt of trinitro- $m$-cresol, the curve was calculated

TABLE 2. Experimental data and association constant for the reaction of tribenzylamine and trinitro-m-cresol in benzene

\begin{tabular}{|c|c|c|c|c|c|c|}
\hline \multirow{3}{*}{$\begin{array}{l}\text { Initial con- } \\
\text { centration of } \\
\text { base a }\end{array}$} & \multicolumn{6}{|c|}{ From measurements at- } \\
\hline & \multicolumn{5}{|c|}{$400 \mathrm{~m} \mu$} & \multirow{2}{*}{$\frac{345 \mathrm{~m} \mu}{K \times 10^{-2}}$} \\
\hline & $a_{s} \mathrm{~b}$ & {$[S] \times 10^{5} \mathrm{c}$} & {$[\mathrm{A}] \times 10^{5} \mathrm{o}$} & {$[B] \times 10^{5} \mathrm{c}$} & $K \times 10^{-2}$ & \\
\hline $0 \ldots$ & 0.0090 & & & & & \\
\hline $1 \ldots$ & .0177 & 0.1273 & 4. 873 & 4. 873 & ${ }^{*} 5.36$ & ${ }^{*} 4.24$ \\
\hline $2 \ldots$ & .0241 & .2209 & 4.779 & 9.779 & $* 4.73$ & $* 4.98$ \\
\hline $4 \ldots \ldots$ & .0367 & .4052 & 4.595 & 19. 60 & ${ }^{*} 4.49$ & ${ }^{*} 4.79$ \\
\hline $10 \ldots$ & .0706 & .9012 & 4.099 & 49. 10 & 4.48 & 4. 54 \\
\hline $20 \ldots$ & .1129 & 1. 520 & 3.480 & 98.48 & 4. 44 & 4. 47 \\
\hline $30 \ldots$ & .1439 & 1. 974 & 3.026 & 148.0 & 4. 41 & 4. 46 \\
\hline $40 \ldots$ & .1726 & 2. 393 & 2. 607 & 197.6 & 4. 65 & 4. 70 \\
\hline $50 \ldots$ & .1864 & 2. 595 & 2. 405 & 247.4 & 4. 36 & 4. 46 \\
\hline $60 \ldots \ldots$ & .2069 & 2. 895 & 2.105 & 297.1 & 4. 63 & 4. 59 \\
\hline $70 \ldots$ & .2161 & 3. 030 & 1.970 & 347.0 & 4. 43 & 4. 55 \\
\hline $80 \ldots$ & .2277 & 3. 200 & 1.800 & 396.8 & 4. 48 & 4. 24 \\
\hline $90 \ldots$ & .2366 & 3. 330 & 1.670 & 446.7 & 4. 46 & 4. 49 \\
\hline $100 \ldots$ & .2441 & 3. 440 & 1.560 & 496.6 & 4. 44 & 4. 30 \\
\hline $200 \ldots$ & .2924 & 4. 146 & 0.854 & 995.8 & ${ }^{*} 4.88$ & $* 4.77$ \\
\hline $300 \ldots$ & .3072 & 4. 363 & .637 & 1,496 . & $* 4.58$ & *4. 20 \\
\hline $400 \ldots$ & .3197 & 4. 546 & .454 & 1,995 . & ${ }^{*} 5.02$ & ${ }^{*} 4.58$ \\
\hline $500 \ldots$ & . 3270 & 4. 652 & .348 & 2,496 . & *5.36 & ${ }^{*} 4.80$ \\
\hline $600 \ldots$ & .3279 & 4. 666 & .334 & 2,996 . & $* 4.66$ & ${ }^{*} 4.23$ \\
\hline \multirow[t]{2}{*}{$\begin{array}{l}\text { Limiting } \\
\text { value } . .\end{array}$} & \multirow[t]{2}{*}{$\mathrm{d}(.3507)$} & \multirow[t]{2}{*}{ 5. 000} & \multirow[t]{2}{*}{-..-- } & -...-. & -..- & -..- \\
\hline & & & & A verage_- & e 4.48 & e 4.48 \\
\hline
\end{tabular}

${ }^{a}$ Expressed as molar equivalents of $5 \times 10^{-5}-M$ trinitro- $m$-cresol.

b A verage of 10 values.

- In moles per liter of solution.

d Calculated as explained in the text.

- Values marked with an asterisk are excluded from the average. from the transmittancy curves for $5 \times 10^{-5}-M$ trinitrocresol and for its mixtures with 20 and 100 molar equivalents of tribenzylamine and the calculated limiting value of $a_{s}$ at $400 \mathrm{~m} \mu$ (used in the computation of $K_{\text {assoc. }}$ ). The quotient, [ $a_{s}$ (salt) $a_{s}$ (acid) $] /\left[a_{s}\right.$ (mixture) $-a_{s}$ (acid) $]$ should be the same for all wavelengths. Two sets of values for the absorbancy versus wavelength were calculated for the salt, one set based on the absorbancy values for the solution that contained 20 molar equivalents of tribenzylamine and the other set based on the values for the solution with 100 molar equivalents of tribenzylamine. The final curve was computed from the average of the two sets of values. The computed curve (dotted line marked $S$ in fig. 4) intersects the three experimentally determined curves at their isosbestic point, which is near 300 $\mathrm{m} \mu$, and is probably correct within \pm 1 percent.

\section{Discussion}

So far as we are aware, the association constants given in section IV are the first values for solutions of acids and bases in benzene to be measured with precision by the spectrophotometric method. In an earlier publication [1], provisional values were given for the association of the indicator acid, bromophthalein magenta, with five different bases in benzene. These values were determined under less favorable experimental conditions but are probably close to the correct values. Nearly 20 years ago, Weissberger and Fasold [11] attempted to measure spectrophotometrically the extent of salt formation in benzene and chloroform solutions of $p$-dimethylaminoazobenzene (methyl yellow) and trichloroacetic acid. The values for $K$ were not constant and showed a trend. Hantzsch had previously emphasized the tendency toward salt formation in an inert solvent as the most logical measure of the strength of acids, and had estimated the relative strengths of several acids by semiquantitative measurements of the extent to which they formed colored salts with basic indicator dyes $[12,13]$. Carboxylic acids are known to be dimerized in inert solvents and, according to Hantzsch, sulfonic acids are associated in chloroform [14]. In titrating acids conductimetrically with aliphatic amines in benzene, Maryott observed unusual inflections in the curves for the titration of trichloroacetic and camphorsulfonic acids that were not present in the curves for the titration of picric 
acid. The peculiarities were interpreted in terms of a reaction between salt and acid, leading to the formation of a complex anion [15]. In all probability, the reaction studied by Weissberger and Fasold was complicated by similar effects.

Maryott has measured the dipole moment at $30^{\circ} \mathrm{C}$ of tribenzylammonium picrate in benzene. The value, $12.0 \times 10^{-18} \mathrm{esu}$, is substantially the same as that found previously for the picrates of more strongly basic amines $[16,17]{ }^{6}$ From the variation of dielectric constant with concentration, the constant for the dissociation of tribenzylammonium picrate into tribenzylamine and picric acid in benzene was determined at $30^{\circ}$ and $40^{\circ}$ $\mathrm{C}$ [17]. On the assumption that the change in enthalpy $(\Delta H)$ is constant over the limited range of temperature involved, the value calculated for $K_{N}$ at $25^{\circ} \mathrm{C}$ is $5.58 \times 10^{-5}$. The corresponding value for the dissociation constant expressed in terms of moles per liter rather than mole fraction is $6.25 \times 10^{-4}$. The reciprocal of this value, $K_{\text {assoc. }}$, is $1.60 \times 10^{3}$, or almost exactly the value obtained by the spectrophotometric method.

The extent of ionic dissociation of picric acid and trinitro- $m$-cresol in water and in absolute methyl and ethyl alcohols has been measured by Goldschmidt and his collaborators. The two acids were found to be highly ionized and of practically equivalent strength in water, in agreement with earlier work of Kendall [21, 22]. Differences were found, however, in the strengths of the two acids in alcoholic solutions. For picric acid, the thermodynamic ionization constants found were $1.547 \times$ $10^{-4}$ in methyl alcohol [23] and $8.32 \times 10^{-5}$ in ethyl alcohol [24]. ${ }^{7}$ For trinitro- $m$-cresol, the respective values were $3.47 \times 10^{-5}[21]$ and $1.59 \times 10^{-5}[24]$. The results for methyl and ethyl alcohol solutions indicate a lower acidic strength for trinitro-mcresol, in agreement with our results for benzene solutions.

On theoretical grounds, this difference in the strength of the two acids could have been predicted. The introduction of a nitro group into the phenol molecule is most effective in increasing the acidic strength when the substituent is in the ortho or para position. This indicates that resonance with quinoid structures is involved, as well as electrostatic interactions between the ionizable

\footnotetext{
6 The values found for tetraalkylammonium picrates in benzene are of the order $18 \times 10^{-18} \mathrm{esu}$ at $25^{\circ} \mathrm{C}$ [18 to 20 ].

7 The value $6.23 \times 10^{-5}$ has been reported for picric acid in butyl alcohol [25].
}

proton and the dipole moment of the nitro group [26 to 28]. In picric acid, with two nitro groups ortho and one para to the $-\mathrm{OH}$ group, both the electrostatic and the resonance effects operate in the same direction, thus accounting for its high strength. If resonance with quinoid structures is prevented from occurring, only the electrostatic effect of nitro groups remains. Wheland and coworkers have shown that in $p$-nitrophenol the introduction of methyl groups ortho to the nitro group causes a diminution in acidity. It is assumed that such groups cause the nitro group to be twisted out of the plane of the benzene ring, thereby preventing quinoid resonance. This effect, which is termed "steric inhibition of resonance," has been observed by other investigators, for bases as well as for acids. The lower acidic strength of trinitro- $m$-cresol as compared with picric acid can be explained by similar arguments, for the two acids differ in structure by the presence in trinitro$m$-cresol of a methyl group adjacent to the para and one of the ortho nitro groups. In this connection, it is interesting to recall that the absorption curve for trinitro- $m$-cresol in benzene was found to be shifted a little - toward shorter wavelengths - from the position of the curves for picric acid. (See p. 600.) This difference is in harmony with current theories of absorption spectra, according to which the energy of excitation is lower for a molecule in which there is resonance among various structures, and hence spectral absorption occurs at longer wavelengths than if there were no resonance. Partial inhibition of resonance should lead to an opposite effect. Remington [29] and several others have observed such effects.

The discovery that acids that appear equally strong in water show differences in acidity in other solvents is not new. Some of the earliest work in this field was by Hantzsch, who showed, for example, that perchloric acid, the halogen acids, nitric acid, and sulfonic acids - all of which appear equally strong in water-exhibit marked differences in intrinsic acidity when comparisons are made in solvents such as chloroform and ether [14]. Numerous workers have pointed out the advantage of working with aprotic solvents, but the use of such media to date has largely been limited to studies of the catalytic effects of different acids and bases.

The intrinsic strength of an acid or base can be 
determined only by the study of a reaction that is reversible to a measurable degree. In water, the ionization of an acid-which is customarily formulated as $\mathrm{HX}+\mathrm{H}_{2} \mathrm{O} \rightleftharpoons \mathrm{H}_{3} \mathrm{O}^{+}+\mathrm{X}^{-}$-goes practically to completion when the acid, HX, relinquishes its proton readily. The great excess of the base, $\mathrm{H}_{2} \mathrm{O}$, is partly responsible for the completeness of the reaction. The strength of the acid can be measured only when the relative strengths of the acid and the base and their relative proportions in various mixtures are such that the reaction is only partly complete.

The results reported in this paper show that the combination of tribenzylamine with picric acid or trinitro- $m$-cresol would be unsuitable for titrimetric analyses. It is an advantage, however, for the determination of relative strengths that the reactions do not proceed to completion. Tables of relative acidic and basic strengths similar to the electromotive series of metals can eventually be constructed when more data are available, but much work remains to be done.

\section{References}

[1] M. M. Davis and P. J. Schuhmann, J. Research NBS 39, 221 (1947) RP1825.

[2] M. M. Davis, P. J. Schuhmann, and M. E. Lovelace, J. Research NBS 41, 27 (1948) RP1900.

[3] Terminology and symbols for use in ultraviolet, visible, and infrared absorptometry, NBS Letter Circular LC857 (May 19, 1947).

[4] M. E. Maclean, P. J. Jencks, and S. F. Acree, J. Research NBS 34, 271 (1945) RP1643.

[5] J. N. Brønsted and R. P. Bell, J. Am. Chem. Soc. 53, 2478 (1931).

[6] G. E. Hilbert, O. R. Wulf, S. B. Hendricks, and U. Liddel, J. Am. Chem. Soc. 58, 548 (1936).
[7] M. M. Davis, E. A. McDonald, and P. J. Schuhmann, unpublished data.

[8] C. A. Kraus, J. Phys. Chem. 43, 231 (1939).

[9] C. A. Kraus and R. M. Fuoss, J. Am. Chem. Soc. 55, 21 (1933).

[10] R. M. Fuoss and C. A. Kraus, J. Am. Chem. Soc. 55, 3614 (1933).

[11] A. Weissberger and K. Fasold, Z. physik. Chem. [A] 15\%, 65 (1931).

[12] A. Hantzsch, Z. Elektrochem. 29, 221 (1923); 30, 194 (1924).

[13] A. Hantzsch and W. Voigt, Ber. deut. chem. Ges. 62, 975 (1929).

[14] A. Hantzsch, Ber. deut. chem. Ges. 60, 1933 (1927).

[15] A. A. Maryott, J. Research NBS 38, 527 (1947) RP1794.

[16] A. A. Maryott, J. Research NBS 41, 1 (1948) RP1896.

[17] A. A. Maryott, J. Research NBS 41, 7 (1948) RP1897.

[18] G. S. Hooper and C. A. Kraus, J. Am. Chem. Soc. 56, 2265 (1934).

[19] J. A. Geddes and C. A. Kraus, Trans. Faraday Soc. 32, 585 (1936).

[20] P. Mortier, Wis. Natuurkund. Tijdschr. 10, 114 (1941).

[21] H. Goldschmidt, E. Marum, and L. Thomas, Z. physik. Chem. 129, 223 (1927).

[22] J. Kendall, Proc. Roy. Soc. (London) [A] 85, 200 (1911).

[23] H. Goldschmidt and H. Aarflot, Z. physik. Chem. 117, 312 (1925).

[24] H. Goldschmidt, E. Marum, and L. Thomas, Z. physik. Chem. 132, 257 (1928).

[25] R. B. Mason and M. Kilpatrick, J. Am. Chem. Soc. 59, 576 (1937).

[26] F. H. Westheimer, J. Am. Chem. Soc. 61, 1977 (1939).

[27] G. W. Wheland, The theory of resonance and its application to organic chemistry, p. 172 (John Wiley \& Sons, Inc., New York, N. Y., 1944).

[28] G. W. Wheland, R. M. Brownell, and E. C. Mayo, J. Am. Chem. Soc. 70, 2492 (1948).

[29] W. R. Remington, J. Am. Chem. Soc. 67, 1838 (1945).

Washington, January 29, 1948. 